\title{
Meeting report of the 74th Congress of the Japanese Gastric Cancer Association
}

\author{
Keitchi Yoshino, Kosei Hirakawa, Toshifusa Nakajima, Shigeaki Yoshida, Nagahiro Saijo, \\ Yoshihiko Maehara, Yu Sakata, Hisakazu Yamagishi, Kimiya Takeshita, Hiroyuki Ono, Yuko Kitagawa, \\ and Seigo Kitano
}

\section{Introduction}

The 74th Congress of the Japanese Gastric Cancer Association (JGCA), held at Tokyo International Forum from February 7-9, 2002, was attended by 1200 participants. The congress president, K. Yoshino of Keio University, adopted the slogan "Let's attack gastric cancer widely: from Gastric Cancer Association to Gastric Association!" and invited papers related not only to gastric cancer but also to gastric physiology and peptic diseases.

A total of 406 papers were presented, covering, as expected, a wide range of gastric diseases. Besides the regular sessions, a special conference was organized to highlight Helicobacter pylori to discuss its etiological role in gastric diseases, recent epidemiological trends, and to present the status of intervention trials. The conference was followed by a meeting to remember the late Dr. T. Kito, former director of Aichi Cancer Center.

Discussions on some of the main topics of the congress are summarized below by a chairperson of the relevant session.

\section{Symposium 1. Present achievement of molecular biology for gastric cancer and its future (chaired by K. Hirakawa, Osaka City Medical University, and Y. Takahashi, Kanazawa University)}

Recent developments of molecular biology in basic research for gastric cancer are a great surprise and hope for us. However, their clinical application is still unclear, and this is a difficult question. In this session, the present achievement of molecular biology for the diagnosis and therapy of gastric cancer and its perspectives were discussed.

Offprint requests to: K. Yoshino, Keio University Faculty of Nursing and Medical Care, 4411 Endo, Fujisawa, Kanagawa 252-0816, Japan
N. Nishimoto and colleagues from Hiroshima University, evaluated the significance of molecular markers such as c-erb-B2, c-met, K-sam, proliferating-cell nuclear antigen (PCNA), p53, vascular endothelial growth factor (VEGF) and matrix metalloproteases (MMPs) compared with clinicopathological factors such as stage, depth, and lymph node metastasis by multivariate analysis. They reported that K-sam, c-met, lymph node metastasis, depth, and stromal types were independent factors influencing survival, and that a combination assay of these molecular markers and clinicopathological factors was more significant for survival than single assays and determinations of each factor.

T. Yasutake and colleagues from Nagasaki University, summarized their achievements in the application of flow cytometry (FCM), fluorescence in situ hybridization (FISH), and comparative genomic hybridization (CGF). They reported that the upregulation of some chromosomes, such as no. 7 and no. 17, was related to lymph node metastasis and proliferation.

M. Inomata and colleagues from Oita Medical University, studied whether we can use anti-Her2 antibody, which is now clinically applied for human breast cancer, for gastric cancer treatment. They reported that tumors with upregulation of Her 2 accounted for $21 \%$ of gastric cancers, and were significantly correlated with shorter survival and liver metastasis. Moreover, most tumors with upregulation of Her2 also showed its upregulation in metastatic sites. From these results, they suppose that we can use anti-Her2 antibody for Her2-positive gastric cancer.

M. Mori and colleagues from Chiba University, studied the construction and gene expression profiling of cDNA microarrays for human gastric cancer. They reported that there were some genes which showed different expression in tumor and normal cells, and suggested that they can apply these results for the diagnosis and therapy of gastric cancer. 
M. Yashiro and colleagues from Osaka City University, studied the molecular mechanism of scirrhous gastric cancer, which is one of the most obstinate cancers, and the application of knowledge of this mechanism for therapy. They reported that fibrous tissues surrounding cancer cells promoted the proliferation of scirrhous cancer specifically by a growth-factor-like protein, which was identified by their basic research. They also showed that Tarnilast, an agent with anti-proliferative action on fibrous tissues, inhibited the growth of scirrhous cancer both in vitro and in vivo.

N. Boku and colleagues from the National Cancer Center East, studied biological markers such as VEGF, p53, bcl-2, gluthathione $S$ transferase-p (GST-p), thymidilate synthase (TS), and dihydropyrimidine dehydrogenase (DPD) as predictors of the outcomes of several chemotherapies for gastric cancer. They reported that there were significant correlations between some markers and the response rate and/or survival of chemotherapies that included cisplatin (CDDP). They summarized their findings, noting that the expression of VEGF may be useful for the selection of chemotherapy for gastric cancers.

In summary, molecular biology has been playing an increasingly important role not only in basic but also in clinical research for gastric cancer. Its clinical application will surely be beneficial for gastric cancer patients in the future (reported by Y. Takahashi).

\section{Special session 1. International discussion on the treatment guidelines for gastric cancer (chaired by T. Nakajima, Cancer Institute Hospital)}

Treatment guidelines (GLs) in the oncology field are still new topics in Japan, although, for more than 10 years, they have been generated, distributed, and accepted in the daily practice of various cancer treatments in Western countries. The Japanese Gastric Cancer Association has just published treatment GLs for gastric cancer for professionals in March, and those for general use in December 2001. This international discussion sought to ascertain the present status of GLs in Western countries, to compare them with those in Japan, and to discuss various related issues arising in daily practice.

In the first part of the session, the development process and present status of GLs in Japan was introduced by T. Nakajima from the Cancer Institute Hospital, Tokyo. The United States GLs were introduced by M. Sasako from the National Cancer Center, Tokyo, followed by a supplemental explanation by M.F. Brennan, President of the International Gastric Cancer Association (IGCA), Memorial Sloan-Kettering Cancer Center, New York. Europe an GLs were introduced by E. Santoro of the Regina Elena National Cancer
Center, Rome, and President Elect of the International Gastric Cancer Association. The expected influences of GLs on the daily practice of gastric cancer treatment, their influence on social aspects, including financial policies and law suits, and the response of patients to GLs were reported by T. Takahashi of the Tokyo Metropolitan Komagome Hospital. Finally, M. Kitajima of Keio University discussed the basic issues of GLs: how to develop GLs according to the principle of evidencebased medicine (EBM), how to establish EBM, and how to collect high-level evidence for the treatment of gastric cancer.

In the latter half of the session, all speakers participated in a discussion on the following topics: (1) what were the changes seen before and after the introduction of GLs? (2) Did guidelines improve the quality and the results of treatment? (3) What are the differences in GLs between Japan and Western countries? (4) Are the Japanese guidelines acceptable in Western countries?

The discussion revealed that the purpose of GLs is quite different between Japan and Western countries, especially in the United States, where the major goal of GLs is to reduce medical expenses in the health insurance system, and GLs have a great impact on daily practice in the United States. Italy has, as yet, no GLs for gastric cancer such as those in the United States, Canada, and Japan, and the selection of treatment depends on the practicing doctors. Extrapolation of one GL to other countries seems to be difficult because of differences in medical practice in each country. For example, D2 dissection is the standard procedure for locally advanced gastric cancer in Japan and some other Asian countries, but it might be unacceptable in some Western countries from the cost-benefit point of view. Prospective randomized trials are recommended to establish high-level evidence for better selection of treatment modalities in daily practice. Although proper evaluation of GLs will take time in Japan, they are necessary for the standardization of the appropriate daily practice of gastric cancer (reported by $\mathrm{T}$. Nakajima).

\section{Special session 2. What do we learn from long} survivors of metastatic gastric cancer treated with systemic chemotherapy? (chaired by S. Yoshida, National Cancer Center; S. Saji, Gifu University; and H. Watanabe, Niigata University)

This program attempted to clarify the characteristics of long survivors among those treated with systemic chemotherapy for their metastatic disease. Y. Hirono, Fukui Medical University, presented an interesting case of a patient with type 4 gastric cancer with simultaneous bone metastasis who died of heart failure without any 
recurrences 9 years and 8 months after surgery. In this particular case, metastasis was limited to only the lumbar vertebra and pelvic bone, and the patient underwent total gastrectomy. Setting aside the controversy in regard to surgical indications, the metastatic lesions responded to postoperative chemotherapy (FAM followed by uracil plus futrafur [UFT]) with radiation, and completely disappeared finally. H. Watanabe, the co-chairman, commented, from the viewpoint of a pathologist, that the characteristic histological features of cancerous tissue found in the resected specimen, i.e., less cellular atypism, may explain the long survival, due to the less invasive behavior, and he suggested that surgery for such a case, i.e., a patient showing less atypical poorly differentiated type (por 2) could be feasible even in the presence of metastatic disease, unlike conventional types.

As to peritoneal dissemination, H. Fujiwara of Iwate Medical University presented his results of intraperitoneal chemotherapy with CDDP with intravenous administration of 5-fluorouracil (5-FU) followed by a standard methotrexate (MTX)-5-FU regimen for those who underwent surgery but were noncurable due to peritoneal dissemination. In his series of 22 patients, the 2 -year survival rate was not so high, at $14 \%$, but 1 of them has survived for more than 8 years and 6 months (still living). In this patient, complete response had been confirmed by a second-look operation performed 16 months after the treatment. Also, H. Inoue of Hiroshima University, presented a report of a patient, still alive, who has survived for more than 10 years after the recurrence. In this paitent, malignant ascites was confirmed 3 months after the surgery, without any other recurrences, and this was treated mainly with intraperitoneal administration of OK-432 (a nonspecific immune-activator) and mitomycin C. Up to the present, the ascites has been controlled well, although the patient has had episodes of rectal stricture and subsequent hydronephrosis due to the presence of localized dissemination in recent years. A. Katayama of Tokyo Metropolitan Komagome Hospital, examined the survival rate of 178 patients who underwent absolutely noncurative surgery. He showed that $4(2.2 \%)$ of the 178 patients survived for more than 5 years, and the noncurative factors in these 4 were P0CY1 (macroscopic $\mathrm{P} 0$ but positive for cancer cells in cytology) in 3 patients and the presence of cancer in the surgical margin in 1 patient. His series included 15 patients in whom the noncurative factors were limited to P0CY1, and 4 of them showed any recurrences during the follow-up period ( 3 survived for more than 5 years and the fourth died of cerebral bleeding). He therefore concluded that P0CY1 should be discriminated from other factors for noncurative status. In the discussion however, he had a question in that his results were different from the general understanding that patients with P0CY1 have a poor prognosis, similar to that for P1. Then he stressed the importance of intensive adjuvant chemotherapy.

As to the prognostic factors, K. Aoyagi of Kurume University, reported the results of a retrospective analysis of 637 patients in stage IV, consisting of 396 surgical and 241 nonsurgical patients. In this series, there were $12(1.9 \%) 5$-year survivors (10 surgical and 2 nonsurgical patients). In the 396 surgical patients, those who received adjuvant chemotherapy had a better prognosis than the others (nonrandomized comparison), and those without $\mathrm{P}$ - and $\mathrm{H}$-factors, in addition to a limited number of metastatic nodes, also showed better survival than the others. From the database of the Japan Clinical Oncology Group (JCOG) trials of systemic first-line chemotherapy for patients with inoperable metastatic disease $(n=497)$, A. Ohtsu, National Cancer Center Hospital East, showed that their 2- and 5-year survival rates were $8 \%$ and $2 \%$, respectively. He presented the results of multivariate analysis of these 497 patients, indicating that performance status, number of metastatic sites, and macroscopic type could be recognized as prognostic factors, and also demonstrated that the macroscopic appearance of nonscirrhous type, limited metastatic site of N4, and history of gastrectomy were significantly dominant in 5-year survivors $(n=9)$ in their series.

The above presentations may indicate the following conclusions: (1) even with metastatic disease, 5-year survival is possible in around $2 \%$ of patients. (2) Long survivors show chemoresponse to some degree. (3) Metastatic organs (sites) are extremely limited in long survivors, and surgery for the primary lesion in such patients may contribute to their survival (reported by S. Yoshida).

Panel discussion 1. Role of drug sensitivity tests in the treatment of gastric cancer (chaired by N. Saijo, National Cancer Center Hospital, and N. Tanigawa, Osaka Medical College)

This panel discussion was organized to evaluate the role of drug sensitivity tests in the selection of effective drugs for individualized chemotherapy of gastric cancer. Many surgeons are interested in the use of drug sensitivity tests, because they can obtain tumor specimens by themselves.

Seven presentations were scheduled, followed by a general discussion. There are numerous problems with the currently available drug sensitivity tests, including variations in methods, low success rates, differences in pharmacokinetics/protein binding/metabolism, and variations in the definition of drug sensitivity. In addition to the speakers who used the MTT assay (W. 
Ichikawa, Saitama Medical School; M. Iwahashi, Wakayama Medical University; B. Nakata, Osaka City University), the four other speakers (T. Nishida, Osaka University; M. Terashima, Iwate Medical College; H. Shinohara, Osaka Medical College; T. Kubota, Keio University) used different drug sensitivity tests, and the validation of these methods has not been completed. This is a serious problem for the development of a randomized controlled trial to demonstrate the superiority of drug selection based on drug sensitivity tests.

Another trend was the use of molecular markers to determine drug sensitivity (T. Nishida, Osaka University; W. Ichikawa, Saitama Medical College; T. Kubota, Keio University). In chemotherapy regimens for stomach cancer, fluorinated pyrimidines are the major agents; the metabolic pathways of these agents include metabolizing enzymes such as dihydropyrimidine dehydrogenase (DPD), thymidylate synthase (TS), and thymidine phosphorylase (TP), which are considered to be determinants of chemosensitivity. In the panel discussion, the majority of participants considered TS to be a prognostic factor and DPD to be a determinant of chemosensitivity.

The points of general agreement were as follows: (1) there is a good correlation between the overexpression of molecular markers and drug sensitivity, and (2) the combined use of molecular tests and drug sensitivity tests increases the physician's ability to predict a patient's resistance to anticancer drugs. The majority of panelists gave optimistic presentations and concluded that drug sensitivity tests could contribute to the individualization of chemotherapy regimens for gastric cancer. However, no data have been obtained confirming that the selection of anticancer drugs based on the drug sensitivity tests can provide better treatment results. Nevertheless, drug sensitivity tests have been used as items of advanced medical technology at five institutions, and some researchers are trying to obtain medical insurance coverage for drug sensitivity tests. However, the data for drug sensitivity tests seem too immature for these steps to be justified. Positive data obtained in randomized clinical trials are essential for the inclusion of drug sensitivity tests in cancer treatments in clinical practice (reported by N. Saijo).

Panel discussion 2. The therapeutic significance of oral fluoropyrimidines in gastric cancer (chaired by Y. Maehara, Kyushu University, and T. Sasaki, Tokyo Metropolitan Komagome Hospital)

Oral fluoropyrimidines, which hold the most important position in chemotherapy on an outpatient basis for gastric cancer, are widely used for patients with advanced disease and as adjuvant chemotherapy. TS-1, in particular, achieved a response rate of $45 \%$ in gastric cancer patients in a phase II study and has been drawing attention as a key drug for gastric cancer. Nine reports at this symposium, all on subjects related to TS-1, presented updated basic and clinical evidence.

Sensitivity to oral fluoropyrimidines, mainly TS-1, and related factors: two reports

H. Usuki of Kagawa Medical University, reported that, in a study of 80 postoperative gastric cancer patients, both UFT and TS-1, which are DPD-inhibitory fluoropyrimidines (DIF), were useful in the treatment of patients with low DPD activity, and that TS-1 was especially useful in patients with high DPD activity. $\mathrm{H}$. Fujiwara of Iwate Medical University, who conducted a basic experiment with nude mice, reported on the selection of indications for oral fluoropyrimidines in view of TS, DPD, and TP enzyme activities.

\section{Clinical results of treatment with TS-1 alone: five reports}

H. Cho of the Kanagawa Cancer Center; H. Kawai of the National Cancer Center Hospital East; and K. Yoshida from Hiroshima University, reported on the clinical results of treatment of gastric cancer with TS-1 alone. Cho reported a survival benefit among patients who received longterm administration of TS-1. Kawai reported that patients who received an initial dose of TS-1 showed a 2-year survival rate of $35 \%$, a rate indicating high efficacy of TS-1. Yoshida emphasized that treatment with TS-1 alone produced better results than treatment with a low dose of cisplatin plus 5-FU at his facility. Yoshida also indicated the possibility of personalized chemotherapy based on an efficacy prediction model using a statistical technique. Table 1 summarizes their results.

H. Imamura from the Sakai Municipal Hospital, reported on the results of a clinical study of the use of TS-1 alone for cur- $\mathrm{C}$ gastric cancer and suggested a possible modified schedule to relieve adverse reactions. T. Kinoshita from the National Cancer Center Hospital East, reported on a pilot study of preoperative chemotherapy with TS-1 for scirrhous gastric cancer. The Japan Clinical Oncology Group (JCOG) is currently carrying out a phase II study of TS-1 preoperative chemotherapy for scirrhous gastric cancer.

Table 1. Treatment results of 3 papers on TS-1

\begin{tabular}{lccc}
\hline & No. of patients & Response rate & MST (d) \\
\hline Cho & 69 & $38 \%$ & 220 \\
Kawai & 51 & $39 \%$ & 337 \\
Yoshida & 27 & $44 \%$ & -
\end{tabular}

MST (d), Median survival time (days) 


\section{TS-1 therapy combined with other drugs: two reports}

I. Takahashi, Kyushu University, reported the results of a study of the antitumor effect and toxicity of combination chemotherapy of TS- 1 and docetaxel with nude rats. An increased antitumor effect was demonstrated in the slight toxicity range. H. Baba from the National Kyushu Cancer Center, reported the therapeutic results for advanced gastric cancer patients treated with a combination of TS- 1 and cisplatin. An assessment of nine evaluable cases showed a response rate of $56 \%$. Baba stated that this combination therapy could be the first choice for advanced recurrent gastric cancer or that it could be suitable for preoperative chemotherapy.

Finally, N. Kaibara of Tottori University, gave a special speech and reported on an investigation of the relationships between the prognoses for patients who received oral fluoropyrimidines as postoperative adjuvant chemotherapy and 5-FU metabolic and catabolic enzymes. He reported on some patients who responded to TS-1 and also described the prospects for the future of TS-1 chemotherapy (reported by Y. Maehara).

\section{Workshop 1. Clinical pathways for gastric cancer} patients (chaired by Y. Sakata, Misawa City Hospital, and A. Nashimoto, Niigata Cancer Center Hospital)

Clinical pathways (CPs) are now being introduced for the standardization of medical practice, shortening of hospital stays, and quality control in medicine. This workshop attempted to demonstrate CPs for gastric cancer patients, and to underline the importance of these CPs. As the keynote address, T. Konishi of Kanto Medical Center NTT E.C. Tokyo, presented the importance and advantages and disadvantages of CPs, such as the shortening of hospital stay, recognition of paramedical staff contribution, and patient satisfaction due to CPs, and then called upon all speakers and the audience to address the questions of outcome and variance. In total, 13 papers were presented, in three categories: CPs for endoscopic surgery, surgical CPs, and evaluation of the efficacy of CPs for patients and staff.

CPs for endoscopic surgery (T. Kato, Niigata Cancer Center Hospital; Y. Kanyama, Nagoya University; K. Shitara, Tokyo Medical and Dental University), such as endoscopic mucosal resection and laparoscopic gastrectomy, reduced costs, shortened hospital stays, and were useful for informed consent and risk management, but variance was frequently observed in very elderly patents and patients who endured a greater extent of resection, such as total gastrectomy.

Two types of surgical CPs were reviewed (A. Kurita, National Shikoku Cancer Center; K. Uchida, Saitama Cancer Center; S. Ohkawa, Cancer Institute Hospital, Tokyo; M. Terashima, Iwate Medical University; A.
Nashimoto, Niigata Cancer Center Hospital; M. Ito, Akita University School of Medicine): one included all methods of gastrectomy, and the other was divided into mainly distal and total gastrectomy. Each speaker emphasized that CPs reduced costs, shortened hospital stays, reduced complications, increased cooperation between medical and paramedical staff, and prevented medical accidents. In their reports, little variance was seen in CPs for distal gastrectomy, whereas a high rate of variance was observed in CPs for total gastrectomy. This high rate of variance was apparently due not only to doctors and hospital systems but was also due to the patients and their complications. We discussed the difficulties and problems with comprehensive CPs encompassing all operative methods, as well as the need to promote better practical procedures clinically for the improvement of CPs.

On the other hand, H. Katai of the National Cancer Center, Tokyo, reported that CPs did not reduced the length of hospital stay or the cost, whereas CPs were positively assessed by both doctors and nurses as good tools for establishing a consensus between them. Using a questionnaire (N. Akutsu, Chiba University, Y. Iwasaki, Tokyo Metropolitan Komagome Hospital), the usefulness of CPs and the satisfaction and anxiety of patients were estimated. In these reports, the anxiety was less and the satisfaction was more in the group treated using CPs than in the group not using them.

We concluded that CPs for gastric cancer are useful tools for good clinical practice and have advantages for patient, and for medical and paramedical staff. In the future, we should establish better CPs and new CPs for chemotherapy and other cancers (reported by Y. Sakata).

Workshop 2. Morbidity and mortality after gastric cancer surgery (chaired by H. Yamagishi, Kyoto Prefectural University of Medicine, and A. Nashimoto, Niigata Cancer Center)

In this workshop, 13 papers were presented to analyze operative morbidity and mortality from various aspects. The authors of the first five papers showed general statistics of surgical complications after gastrectomy in their hospitals. In the next two papers, a surgical scoring system (E-PASS; estimation of physiologic ability and surgical stress) and new molecular markers, such as serum cytokines, systemic inflammatory response syndrome (SIRS) score, and MHC-class II antigen (HLA-DR) were discussed in relation to the operative complications. In five other papers, the authors examined various complications related to specific conditions, such as thoracotomy for tumors invading the esophagus, the presence of pancreatic fistula, the exist- 
ence of peritoneal dissemination, or the advanced age of patients. In the remaining paper, the morbidity and mortality in a randomized controlled trial (RCT) were presented.

The post-gastrectomy morbidity rates reported in the session were around $20 \%$. The rate was lower in women $(18 \%)$ than in men $(23 \%)$ in one paper presented, and was higher in patients over 80 years of age $(30 \%-$ $33 \%)$. Paraaortic lymphadenectomy was associated with higher morbidity (28\%) than standard D2 (21\%) in the RCT.

The rates of operative death within 30 days after standard gastrectomy were all lower than $1 \%$. The operative mortality was high in tumors invading the esophagus $(3.9 \%)$ in one study, but was not increased by extended paraaortic dissection in the RCT $(0.4 \%)$. In papers in which the time trend was examined, both morbidity and mortality rates showed significant decreases in recent years. All authors agreed that anastomotic leak, pancreatic fistula, and respiratory complications are major factors related to operative death.

T. Sano, National Cancer Center Hospital, reported the results of the RCT in which more than 500 patients were recruited by 26 institutions (Japan Clinical Oncology Group [JCO6] Study 9501). They showed that, in these specialized hospitals, paraaortic lymphadenectomy could be performed without increasing the operative mortality. We need to know the prognostic benefits of this procedure to generalize it as a standard operation for advanced gastric cancer (reported by $\mathrm{H}$. Yamagishi).

\section{Workshop 3. Follow-up system after gastrectomy} (chaired by I. Honda, Chiba Cancer Center Hospital, and K. Takeshita, Tokyo Medical and Dental University)

The eight papers presented in this session were classified into three categories: follow-up for recurrence, metachronous cancers, and post-gastrectomy syndromes.

T. Ogawa and colleagues from the National Defense Medical College, evaluated follow-up intervals of endoscopy for diagnosing cancer in the remnant stomach. They found ten cases $(0.6 \%)$ of stump carcinoma arising after gastrectomy in their institution. Early stump carcinomas were all detected by annual endoscopy, while advanced tumors were diagnosed after 2-year or longer intervals.

T. Doi and colleagues from the National Shikoku Cancer Center, studied locally recurrent or multifocal cancers after endoscopic mucosal resection (EMR). They experienced local recurrence in $24(5.2 \%)$ patients, most of which were treated by endoscopic re- resection. Of 44 cases of multifocal cancers, $70 \%$ of the lesions were detected either before or within 1 year after EMR. Metachronous lesions were detected 4-5 years after the initial EMR. Multifocal cancers could be successfully treated by EMR if detected early.

Y. Kakechi and colleagues from Kyushu University, found that positive expression of VEGF in the primary lesion was associated with hematogenous recurrence, while positive expression of p53 was a risk factor for lymphatic metastasis. The serum tumor markers CEA and STN were associated with hepatic and peritoneal recurrence, respectively. Based on these results, they proposed a follow-up system for early detection of recurrence.

S. Ishigami and colleagues from Kagoshima University, presented their follow-up schedule according to the risk of recurrence. They classified the risk of a patient into three grades (low, intermediate, high) based on the findings of micrometastasis, as well as the tumor stage. Their follow-up usually includes determination of tumor markers and abdominal computed tomography (CT), and they alter the intervals according to the risk of recurrence in each patient.

C. Kunisaki and colleagues of Yokohama City University, studied the risk factors and disease-free intervals for various patterns of recurrence, using a multivariate analysis. They found that the peritoneum was the major recurrent site in the early postoperative years, while hematogenous metastasis increased after 2 to 3 years. Lymphatic recurrence showed no specific trend. They proposed a follow-up system based on the risk factors and possible recurrent sites.

H. Saeki and colleagues from Yokohama City University, presented their follow-up system with special emphasis on the quality of life of patients undergoing distal gastrectomy. They found gastritis in 30\% of patients with Billroth-I reconstruction, while it was rare after Roux-en-Y reconstruction or pylorus-preserving gastrectomy. Postoperative gallstones were found in $23 \%$ and $0 \%$ after D2 lymphadenectomy and vaguspreserving lymphadenectomy, respectively. There were no differences in the incidence of bone density disturbance among the three reconstruction methods.

K. Ohta and colleagues from the Cancer Institute Hospital, analyzed the causes of death and the patterns of recurrence in a large number of patients according to the T-stage of the tumors. They proposed, for patients with advanced disease, digital rectal examination or barium enema for the early detection of peritoneal recurrence, in addition to periodical tumor marker determinations and abdominal CT.

K. Arai and colleagues of Tokyo Metropolitan Komagome Hospital, comprehensively discussed the meaning of postoperative follow-up. They showed the timing of onset of post-gastrectomy symptoms such as 
dumping syndrome, reflux esophagitis, and vitamin B12-deficiency anemia. Second malignancies are an important issue, and they suggested the necessity for education of patients and network establishment for periodical health checks (reported by K. Takeshita).

Video symposium 1. To what extent is endoscopic mucosal resection (EMR) for early gastric cancer permissible? (chaired by R. Hirayama, Saitama Medical College; M. Itabashi, Ibaraki Prefectural Central Hospital; and H. Ono, National Cancer Center Hospital)

EMR for early gastric cancer (EGC) was developed in 1983 and now is widely accepted in Japan. In 2001, the Japanese Gastric Cancer Association published the gastric cancer treatment guidelines in which the criteria for EMR were proposed, as follows: intramucosal cancer, and histologically of differentiated type, and smaller than $20 \mathrm{~mm}$, and no ulcerative findings in the lesion. However, many endoscopists seem to consider the criteria as a minimal standard that can be expanded in the future. All speakers in the session agreed that two issues should be addressed for the expansion of EMR indications: the possibility of lymph node metastasis and technical problems.

I. Oda and colleagues from the National Cancer Center Hospital reviewed over 3000 cases of surgically resected EGCs and concluded that in intramucosal, histologically differentiated-type tumors, there were no lymph node metastases either when the tumor had no ulcerative changes, or when it was smaller than $3 \mathrm{~cm}$ with ulcerative changes. M. Ishigooka and colleagues from Kin-Ikyo Chuo Hospital, obtained the same results from their data. With regard to lymph node metastasis, we reached a consensus to follow Oda's proposal.

As for the technical problems, the issue of multifragment resection was first discussed. Multifragment resection for a lesion has been reported to be associated with an increased incidence of local tumor recurrence due to incomplete local control. However, M. Tani and colleagues from Tokyo Medical and Dental University, evaluated their EMRC method and found a low incidence of residual disease by well-planned multifragment resection for lesions smaller than $25 \mathrm{~mm}$. N. Uedo, Osaka Medical Center for Cancer and Cardiovascular Diseases, also focused on this issue and reviewed their 103 cases of single-fragment resection and 20 cases of multifragment resection. He found no significant difference in prognosis between the two groups, although the multifragment group had more local recurrences requiring additional therapy than the single-fragment group. Four other speakers, who usually employ the cutting method for EMR, stated their position that operators should aim to carry out singlefragment resection.

I. Oda introduced the insulation-tipped diathermic knife (IT knife) technique for single-fragment resection of large and ulcerative lesions. He showed that more than $90 \%$ of lesions larger than $30 \mathrm{~mm}$ were removed in a single fragment by this method. M. Ishigooka introduced an ERHSE method and also reported good results with this method.

T. Doi of the National Shikoku Cancer Center Hospital simplified the IT knife technique for "beginners" by improving the cutting device. He emphasized that the IT knife method was good, but that at present it needs some modification to be generalized. H. Imaeda, Keio University, used several techniques, including the IT knife, needle knife, and their "intragastric EMR" for single-fragment resection and presented their good results.

In summary, the present criteria for gastric EMR proposed by the JGCA are suitable for general endoscopists. On the other hand, it seems possible to expand the criteria as for as lymph node metastasis is concerned. We should improve the techniques for generalization and further expansion of the criteria (reported by H. Ono).

Video symposium 2. Sentinel node mapping for gastric cancer (chaired by M. Sasako, National Cancer Center, Tokyo, and Y. Kitagawa, Keio University, Tokyo)

The first possible sites of metastasis via lymphatic drainage from a primary lesion are known as sentinel nodes (SNs). An orderly progression of lymph node metastasis has been well demonstrated for both malignant melanoma and breast cancer over the past decade. However, the feasibility of SN mapping in gastric cancer and its diagnostic reliability are still controversial. In this session, future applications of this technique in modified and less invasive surgery for early gastric cancer (EGC) were demonstrated by video presentation. Technical problems in this field were also extensively discussed.

At the beginning of the session, the definition of the parameters, the detection rate, and the diagnostic sensitivity, to evaluate the validation studies, were clarified and confirmed by M. Sasako of National Cancer Center as a chairperson. Updated data from the institutes presenting the paper in this session were summarized. Although data almost compatible with the initial experience in melanoma and breast cancer were demonstrated, the patient population enrolled was still too limited to validate this concept in gastric cancer. Y. Kitagawa, Keio University, demonstrated the feasibility of radio-guided SN detection in laparoscopic surgery. 
This procedure would be applicable for laparoscopic wedge resection or sleeve resection for EGC without lymph node metastasis. T. Sawada, Osaka City University, reported the technical details of laparoscopyassisted gastric segmentectomy combined with dye-guided SN mapping. M. Hiratsuka from the Osaka Medical Center for Cancer and Cardiovascular Diseases, has established dye-guided SN mapping using indocyanine green, and has already published results showing high detection rates and diagnostic accuracy for cT1N0 gastric cancer. Based on their own results, they have started a single institutional clinical study of local resection for cT1N0 patients with negative SN status. The reliability of the intraoperative evaluation of $\mathrm{SN}$ would be the critical issue in this project. K. Abe of Iwate Medical University, and T of Ichikura, National Defense Medical College, have compared the results of SN mapping using radioactive colloid and blue dye as tracers. They have shown a significant discrepancy of SN distribution using these different tracers and recommended the combined method as a safer procedure to avoid false-negative results. Interference in lymphatic channels by radioactive colloid might be the explanation for the modified migration patterns of blue dye. Y. Takagi from Tokyo Medical University, reported some difficulties in intraoperative radio-guided SN detection because of a "shine-through" effect and residual radioactivity in the liver and small intestine. To overcome these problems, they have introduced a lead plate to avoid the shine-through activity. S. Kinami, from a group at Kanazawa University, has previously published details of the procedure for intraoperative endoscopic lymphatic mapping with vital dye. They have introduced a radio-guided method using $99 \mathrm{mTc}$ phytate combined with vital dye to apply this technique to laparoscopic surgery. Visibility in the dye-guided method and stable accumulation of tracer in the radioguided method were demonstrated as advantages in a combined method. S. Hokita and colleagues from Kagoshima University, have investigated the clinical significance of micrometastasis in EGC. They showed an increased sensitivity for detecting micrometastasis using anti-cytokeratin immunohistochemistry in comparison with conventional H\&E staining. The methodology of the intraoperative detection of micrometastasis is one of the critical issues for the clinical application of modified surgery based on SN status. From the group at Jikei University, H. Nimura introduced infrared ray electronic endoscopy as a tool to improve the visibility of indocyanine green.

Although there are several future applications of SN mapping in individualized and modified gastric cancer surgery, we have to resolve many remaining issues. Multicenter prospective validation studies with sufficient numbers of patients are urgently required to prove the clinical significance of this technology (reported by Y. Kitagawa)

Video symposium 3. Evaluation of laparoscopic gastrectomy for gastric cancer (chaired by T. Aiko, Kagoshima University, and S. Kitano, Oita Medical University)

Since 1991, laparoscopic gastrectomy for gastric cancer has been widely performed in Japan. During the early 1990s, laparoscopic wedge resection for early gastric cancer (EGC) without lymph node metastasis was performed regularly. Due to the development of new techniques and instruments in the field of laparoscopic surgery, laparoscopy-assisted distal gastrectomy (LADG) with $\mathrm{D} 1+\alpha$ lymph node dissection has become popular in managing patients with EGC with a risk of perigastric lymph node metastasis. Recently, some surgeons have been performing LADG with D2 dissection even for advanced gastric cancer. In this video symposium, the procedures and clinical outcomes of these operations were evaluated, and the indications for and usefulness of laparoscopic gastrectomy were discussed.

Y. Otani of Keio University, Tokyo, reported the usefulness of laparoscopic wedge resection for gastric cancer. His group has treated 112 patients with EGC and 60 patients with gastric submucosal tumor by the lesion-lifting method since 1992. The indications for laparoscopic wedge resection are as follows: elevated mucosal carcinoma $(<25 \mathrm{~mm})$, depressed mucosal carcinoma $(<15 \mathrm{~mm})$ without ulceration, and submucosal tumor from 2 to $5 \mathrm{~cm}$ in diameter. This study showed that laparoscopic wedge resection has advantages including the rapid recovery of gastrointestinal function and a short hospital stay. All patients treated with laparoscopic wedge resection have survived without recurrence. They concluded that laparoscopic wedge resection was curative, safe, and useful in patients with EGC and stromal tumors of the stomach.

I. Miyashiro from the Osaka Medical Center for Cancer and Cardiovascular Diseases indicated the clinical significance of sentinel node (SN) biopsy for gastric cancer. They performed SN biopsy in patients with T1 $(n=63)$ or T2 $(n=36)$ gastric cancers by dye injection (indocyanine green). In the $\mathrm{T} 1$ group, $\mathrm{SN}$ biopsy sensitivity and specificity were both $100 \%$. They concluded that SN biopsy could predict lymph node status with high accuracy in patients with T1 gastric cancer and it might be useful for selecting surgical options, including laparoscopic gastrectomy.

H. Nimura of Jikei University, Tokyo, reported the usefulness of SN navigation using infrared ray rigid endoscopy (IRRE) in the treatment of EGC. Fifty-three patients with gastric cancer underwent IRRE-SN navi- 
gation surgery; 45 with conventional, and 8 with laparoscopic gastrectomy. Sentinel nodes were identified in all cases. Eight patients had histologically positive nodes, and these nodes were detected during the operation in six of the eight patients. The high incidence of identification of positive nodes during surgery was achieved only by histological examination with cytokeratin staining. When SN navigation by IRRE is performed, the indications for laparoscopic wedge resection of the stomach may be expanded as follows: mucosal carcinoma $(<30 \mathrm{~mm}$ in diameter $)$ with ulceration scar, well-differentiated carcinoma $(<30 \mathrm{~mm})$ with slight submucosal invasion, and poorly differentiated carcinoma $(<30 \mathrm{~mm})$ with slight submucosal invasion and no ulceration. They concluded that SN navigation with IRRE was acceptable in the treatment of EGC and may expand the indications for laparoscopic wedge resection of the stomach.

N. Shiraishi of Oita Medical University evaluated the usefulness of LADG in a prospective randomized control study. The indication for LADG with $\mathrm{D} 1+\alpha$ dissection is EGC with risk of positive perigastric nodes. Since 1991, their group has performed LADG in 101 patients with EGC. To clarify the clinical advantages of LADG over conventional distal gastrectomy (DG), a randomized control study was carried out. This study demonstrated that LADG has several advantages over conventional DG, including earlier patient recovery, less pain, and less impaired pulmonary function after gastric surgery. To expand the indications for LADG to advanced gastric cancer without serosal invasion, handassisted techniques for D2 dissection were added to LADG (called HLADG). So far, only three patients with gastric cancer have undergone HLADG, but they suggested that the invasiveness of HLADG seemed to be the same as that of LADG, because of low serum Creactive protein levels after operation. They emphasized that LADG was an acceptable treatment for patients with EGC and that HLADG might also be useful for advanced gastric cancer.

S. Tanimura from Osaka City General Hospital evaluated both the clinical outcomes of patients treated by laparoscopic surgery with D2 lymph node dissection for gastric cancer, and the procedure itself. In 133 patients undergoing LADG with D2 dissection, the time to oral intake and flatus and the postoperative hospital stay were significantly shorter than those in 100 pa-tients undergoing conventional DG. There were no differences between the groups in terms of total number of dissected lymph nodes. They suggested that laparoscopic D2 lymph node dissection was technically feasible and less invasive than the conventional method.

I. Uyama of the Fujita Health University, Nagoya, reported on laparoscopic D2 dissection (called en-bloc dissection) for advanced gastric cancer. They demonstrated a comprehensive lymph node dissection that involved taping and complete skeletonization of the common hepatic and proximal splenic arteries, using laparoscopic procedures. Complete laparoscopic D2 lymph node dissection was performed in 20 patients according to the 13th edition of the Japanese classification of gastric carcinoma. This report suggested that their surgical procedure was technically feasible and safe, and can be extended to stage IIIB gastric cancer.

In summary, laparoscopic gastrectomy for EGC has been performed successfully in Japan. When SN is fully accepted in relation to the treatment of EGC, the number of laparoscopic wedge resections will increase. LADG with perigastric lymph node dissection $(\mathrm{D} 1+\alpha)$ was recognized as a safe and useful procedure in managing patients with EGC. Technical standardization and more clinical studies regarding the usefulness of LADG with extended dissection (D2) are needed to accept the application of LADG in the treatment of advanced gastric cancer (reported by S. Kitano). 